\title{
The Convergence of Multi-Level Methods for Solving Finite-Element Equations in the Presence of Singularities
}

\author{
By Harry Yserentant
}

\begin{abstract}
The known convergence proofs for multi-level methods assume the quasi-uniformity of the family of domain triangulations used. Such triangulations are not suitable for problems with singularities caused by re-entrant corners and abrupt changes in the boundary conditions. In this paper it is shown that families of properly refined grids yield the same convergence behavior of multi-level methods for such singular problems as quasi-uniform subdivisions do for $H^{2}$-regular problems.
\end{abstract}

1. The Continuous Problem, Its Discretization and the Multi-Level Method. Using multi-level techniques ([1], [3], [4], [6], [7], [8], [10]), it is possible to solve the large systems of linear equations arising in connection with finite-element methods with an amount of work roughly proportional to the number of unknowns. This property makes multi-level methods at least theoretically superior to all other solution methods, including fast solvers based on FFT-like algorithms which may be directly applied to special problems only.

The convergence of multi-level methods was proved, for example, by Nicolaides [10], Bank and Dupont [4] and Hackbusch [8]. All these proofs assume a certain amount of elliptic regularity of the continuous problem to be solved approximately, and quasi-uniform subdivisions of the domain in finite elements. Assuming $\mathrm{H}^{1+\alpha}$ regularity, such quasi-uniform triangulations and a Jacobi-like smoothing procedure, Bank and Dupont [4] and Hackbusch [8] showed the following result: The rate of convergence of a full iteration step of the multi-level method behaves like $O\left(\mathrm{~m}^{-\alpha / 2}\right)$, uniformly in the number of levels, for a growing number $m$ of smoothing steps per level. In the optimal case $\alpha=1$, the problem has to be $H^{2}$-regular. This means, for example, that the region is not allowed to have re-entrant corners. If this condition is violated, the convergence rate of the multi-level procedure decreases, and, in addition, the approximation properties of the finite-element discretization itself change for the worse because of the presence of singularities in the solution not captured by the quasi-uniform grids. The strongly nonuniform, systematically refined triangulations suitable for these problems are not included in the theory so far.

The aim of the present paper is to fill this gap.

Received August 31, 1982; revised November 16, 1983.

1980 Mathematics Subject Classification. Primary 65N30, 65F10.

(C1986 American Mathematical Society $0025-5718 / 86 \$ 1.00+\$ .25$ per page 
We take the boundary value problem

$$
\begin{aligned}
-\Delta u+u=f & \text { in } \Omega, \\
u=0 & \text { on } \Gamma_{D}, \\
\partial u / \partial n=0 & \text { on } \Gamma_{N},
\end{aligned}
$$

as a prototype of the problems under consideration. $\Omega$ is an open bounded polygonal subset of $\mathbf{R}^{2}$, the boundary of which is subdivided in two parts, $\Gamma_{D}$ and $\Gamma_{N}$, each consisting of finitely many pieces of straight lines. Let $H_{D}^{1}(\Omega)$ be the space of all functions $u \in H^{1}(\Omega)$ with $u=0$ on $\Gamma_{D}$ in the sense of traces. We understand the model problem (1.1) in the usual weak sense: Given a linear continuous functional

$$
f^{*}(v)=\int_{\Omega} f v d x
$$

on $H_{D}^{1}(\Omega)$, we have to find a function $u \in H_{D}^{1}(\Omega)$ with

$$
B(u, \chi)=\int_{\Omega}\left\{\sum_{i=1}^{2} D_{i} u D_{i} \chi+u \chi\right\} d x=f^{*}(\chi)
$$

for all functions $\chi \in H_{D}^{1}(\Omega)$. It is well known that this boundary value problem has a uniquely determined solution depending continuously on the functional $f^{*}$ from the dual space of $H_{D}^{1}(\Omega)$. We want to solve it approximately, using the finite-element method.

By a triangulation $\mathscr{T}$ of $\bar{\Omega}$ we mean a finite collection of closed triangles, the finite elements, the union of which is $\bar{\Omega}$, such that the intersection of each two finite elements is either empty, or a common side, or a common vertex. In this paper we use a family $\mathscr{T}_{0}, \mathscr{T}_{1}, \mathscr{T}_{2}, \ldots$ of triangulations of $\bar{\Omega}$ such that $\mathscr{T}_{j+1}$ is a refinement of $\mathscr{T}_{j}$. That means that each triangle $T \in \mathscr{T}_{j}$ has to be a union of triangles of $\mathscr{T}_{j+1}$. Let $S\left(\mathscr{T}_{j}\right)$ be the space of functions continuous on $\bar{\Omega}$ and linear on the finite elements $T \in \mathscr{T}_{j}$, and let $S_{D}\left(\mathscr{T}_{j}\right)$ be the space of all functions from $S\left(\mathscr{T}_{j}\right)$ vanishing on the boundary piece $\Gamma_{D}$ from (1.1). The spaces $S_{D}\left(\mathscr{T}_{j}\right)$ are subspaces of the solution space $H_{D}^{1}(\Omega)$ of the continuous problem (1.1), (1.3). Thus it is possible to find an approximation $P_{j} u \in S_{D}\left(\mathscr{T}_{j}\right)$ for the exact solution $u \in H_{D}^{1}(\Omega)$ of (1.3) which satisfies the equation

$$
B\left(P_{j} u, \chi\right)=f^{*}(\chi)
$$

for all functions $\chi \in S_{D}\left(\mathscr{T}_{j}\right)$. Because of the equation

$$
B\left(P_{j} u, \chi\right)=B(u, \chi), \quad \chi \in S_{D}\left(\mathscr{T}_{j}\right),
$$

the approximation $P_{j} u$ is a projection of the function $u \in H_{D}^{1}(\Omega)$ onto the subspace $S_{D}\left(\mathscr{T}_{j}\right)$ of $H_{D}^{1}(\Omega)$. Our aim is to solve the linear system (1.4). For this purpose, we use a multi-level method.

To describe the multi-level iteration, we need a new inner product on the finite-element space $S\left(\mathscr{T}_{j}\right)$. Let $\mathscr{N}_{j}$ be the set of nodes of the triangulation $\mathscr{T}_{j}$, that is, the vertices of the triangles of $\mathscr{T}_{j}$. For functions $u, v \in S\left(\mathscr{T}_{j}\right)$ we define the inner product $E_{j}$ on $S\left(\mathscr{T}_{j}\right) \times S\left(\mathscr{T}_{j}\right)$ by

$$
E_{j}(u, v):=\sum_{T \in \mathscr{T}_{j}} \sum_{x \in \mathscr{N}_{j} \cap T} u(x) v(x) .
$$


This inner product induces the norm

$$
\|u\|_{j}:=\left(\sum_{T \in \mathscr{T}_{j}} \sum_{x \in \mathscr{N}_{j} \cap T}|u(x)|^{2}\right)^{1 / 2}
$$

on $S\left(\mathscr{T}_{j}\right)$. If $n_{j}(x)$ is the number of triangles of $\mathscr{T}_{j}$ containing the node $x \in \mathscr{N}_{j}$, we have for $u, v \in S\left(\mathscr{T}_{j}\right)$

$$
\begin{aligned}
& E_{j}(u, v)=\sum_{x \in \mathscr{N}_{j}} n_{j}(x) u(x) v(x), \\
& \|u\|_{j}=\left(\sum_{x \in \mathscr{N}_{j}} n_{j}(x)|u(x)|^{2}\right)^{1 / 2} .
\end{aligned}
$$

The bilinear forms $E_{j}$ represent the smoothing procedure in the multi-level process. They can be replaced by similar positive definite and symmetric bilinear forms, which lead to norms equivalent to (1.9) in an appropriate sense.

First, we define two-level methods. Given a linear function $f^{*}$ on $H_{D}^{1}(\Omega)$, our aim is to find a function $P_{j} u \in S_{D}\left(\mathscr{T}_{j}\right)$ satisfying (1.4) for all $\chi \in S_{D}\left(\mathscr{T}_{j}\right)$. A single iteration step of the two-level method for solving this problem looks as follows:

Let an approximation $u_{0} \in S_{D}\left(\mathscr{T}_{j}\right)$ of $P_{j} u$ be given. First, a correction $d \in S_{D}\left(\mathscr{T}_{j-1}\right)$ from the coarser finite-element space $S_{D}\left(\mathscr{T}_{j-1}\right) \subseteq S_{D}\left(\mathscr{T}_{j}\right)$, satisfying

$$
B(d, \chi)=f^{*}(\chi)-B\left(u_{0}, \chi\right)
$$

for all $\chi \in S_{D}\left(\mathscr{T}_{j-1}\right)$, is computed. Then

$$
u_{1}=u_{0}+d
$$

is expected to be a better approximation of $P_{j} u$. Now further approximations $u_{2}, \ldots, u_{m+1} \in S_{D}\left(\mathscr{T}_{j}\right)$ are computed, using the recursion

$$
E_{j}\left(u_{l}-u_{l-1}, \chi\right)=\omega\left\{f^{*}(\chi)-B\left(u_{l-1}, \chi\right)\right\}, \quad \chi \in S_{D}\left(\mathscr{T}_{j}\right) .
$$

$m$ and $\omega$ are fixed numbers; $\omega$ is determined later. Then $u_{m+1}$ is the result of the full iteration step with $u_{0}$ as starting value.

The linear system (1.12) can be solved very fast and cheaply because of the simple structure (1.8) of the inner product $E_{j}$. If the usual nodal basis of $S\left(\mathscr{T}_{j}\right)$ is used, the matrix of the system (1.12) has diagonal form. Contrary to these smoothing steps, the computation of the correction term $d$ as the direct solution of the linear system (1.10) is expensive. It is necessary to solve a problem analogous to (1.4) on the smaller space $S_{D}\left(\mathscr{T}_{j-1}\right)$. Therefore, true multi-level methods continue the process until reaching the lowest level. They are defined recursively as follows:

For $j=0$, the problem (1.4) is solved by a direct method.

For $j=1$, the problem (1.4) is solved by the described two-level procedure.

For $j \geqslant 2$, the problem (1.4) is solved by a method analogous to the described two-level method, but the solution of the linear system (1.10) is replaced by the approximate solution obtained with $p \geqslant 2$ steps of the $(j-1)$-level method. The starting value is $d_{0}=0$.

If the family of grids is refined in an appropriate way, the convergence of this process can be guaranteed also for problems with strong singularities. 
As usual, our proof of convergence is based on an approximation property of the finite-element spaces and on a smoothing property. But contrary to the proofs cited above, we give these two properties a purely algebraic form, which makes it possible to treat nonuniform families of grids.

The approximation property: There exists a constant $K$ independent of $j \geqslant 1$ with

$$
\left\|u-P_{j-1} u\right\|_{j} \leqslant K\left\|u-P_{j-1} u\right\|_{1}
$$

for all functions $u \in S_{D}\left(\mathscr{T}_{j}\right)$.

The smoothing property: There is a constant $K_{1}$ independent of $j$ with

$$
\|u\|_{1} \leqslant K_{1}\|u\|_{j}
$$

for all functions $u \in S_{D}\left(\mathscr{T}_{j}\right)$.

The norm on the right-hand side of (1.13) and the left-hand side of (1.14) is the energy norm

$$
\|u\|_{1}=\left(\sum_{|\alpha| \leqslant 1} \int_{\Omega}\left|\left(D^{\alpha} u\right)(x)\right|^{2} d x\right)^{1 / 2}
$$

induced by the bilinear form $B$ in (1.3). As we shall see in Section 2, the smoothing property is nearly trivial, whereas the approximation property has a hard analytic background. To prove it, one needs that the family of grids is properly adapted to the possible singularities in the solution of the continuous problem. At this point, we make use of the elliptic regularity theory. Intuitively, the approximation property says that the error between a function from a finite-element space of a given level and its projection onto the finite-element space of the next coarser level is fast oscillating in the sense of the local grid width. This is the reason why it can be damped out by a local relaxation like (1.12) and why multi-level methods are commonly believed to work so well.

Using the approximation property (1.13) and the smoothing property (1.14), we prove in Section 3 that one step of the multi-level iteration reduces the energy norm of the error by a factor behaving like $O(1 / \sqrt{m})$ for a growing number $m$ of smoothing steps per level, uniformly in the number of levels, if the parameter $\omega$ in (1.12) is chosen correctly. This is qualitatively the same behavior as predicted by Bank and Dupont [4] for $H^{2}$-regular problems and agrees with the observations made in numerical computations [5]. The error reduction factor with respect to the energy norm is expressed in terms of the constants from the approximation and the smoothing property.

2. Proof of the Approximation and Smoothing Properties. We begin with the proof of the smoothing property (1.14), which is an easy task if we assume that the interior angles of the triangles in all triangulations are bounded away from zero by a positive constant.

THEOREM 2.1. There is a constant $K_{1}$ independent of $j$ with

$$
\|u\|_{1} \leqslant K_{1}\|u\|_{j}
$$

for all functions $u \in S_{D}\left(\mathscr{T}_{j}\right)$. 
Proof. As the interior angles of the triangles $T \in \mathscr{T}_{j}$ are bounded away from zero, there is a constant $K_{1}$ depending only on this bound and on an upper bound for the diameters of the triangles such that

$$
\sum_{|\alpha| \leqslant 1} \int_{T}\left|\left(D^{\alpha} u\right)(x)\right|^{2} d x \leqslant K_{1}^{2} \sum_{x \in \mathscr{N}_{j} \cap T}|u(x)|^{2}
$$

for all first-order polynomials $u$, hence for all functions $u \in S\left(\mathscr{T}_{j}\right)$. A summation over all triangles $T \in \mathscr{T}_{j}$ gives the desired result.

Note that the proof of Theorem 2.1 is constructive: The constant $K_{1}$ can be taken as the square root of the largest eigenvalue of the local stiffness matrices for the single finite elements. This enables us to choose the parameter $\omega$ in the definition of the two- and the multi-level method correctly; see Theorems 3.1 and 3.2 below.

Conversely, the approximation property states that the norm induced by the inner product $B$ majorizes the norm associated with $E_{j}$ for functions $u \in S_{D}\left(\mathscr{T}_{j}\right)$ orthogonal to $S_{D}\left(\mathscr{T}_{j-1}\right)$ with respect to $B$. To prove this, we have to make an essential assumption on how the triangulations are refined: Let the points $x_{1}, \ldots, x_{n}$ be the vertices of the domain $\Omega$, including the points of abrupt changes from Dirichlet to natural boundary conditions. For each vertex $x_{i}$ we define $\kappa_{i}=1$ if the two sides having the endpoint $x_{i}$ belong either both to $\Gamma_{D}$ or both to $\Gamma_{N}$, and $\kappa_{i}=\frac{1}{2}$ otherwise. Let $0<\theta_{i}<2 \pi$ be the interior angle of $\Omega$ at the point $x_{i}$; because of possible changes in the boundary conditions, the case $\theta_{i}=\pi$ is permitted. Let $\alpha_{i}=$ $\min \left\{1, \kappa_{i} \pi / \theta_{i}\right\}$. Then $\frac{1}{4} \leqslant \alpha_{i} \leqslant 1$ holds. If we have pure Dirichlet or natural boundary conditions, $\alpha_{i}<1$ only holds for re-entrant corners. We now choose coefficients $\gamma_{i}$ with $1-\alpha_{i}<\gamma_{i}<1$ if $\alpha_{i}<1$, and set $\gamma_{i}=0$ if $\alpha_{i}=1$. Define the function $\phi_{\gamma}$ on $\mathbf{R}^{2}$ for $\gamma=\left(\gamma_{1}, \gamma_{2}, \ldots, \gamma_{n}\right)$ by

$$
\phi_{\gamma}(x)=\prod_{i=1}^{n}\left|x-x_{i}\right|^{\gamma_{i}}
$$

where $|x|$ denotes the Euclidean length of the vector $x$. We assume that the family $\mathscr{T}_{0}, \mathscr{T}_{1}, \mathscr{T}_{2}, \ldots$ of triangulations has the following two properties:

If $T$ is a triangle from triangulation $\mathscr{T}_{j}$ and $\phi_{\gamma}$ vanishes nowhere on $T$, the estimate

$$
C_{1} 2^{-j} \phi_{\gamma}(x) \leqslant d(T) \leqslant C_{2} 2^{-j} \phi_{\gamma}(x)
$$

holds for all $x \in T$, with positive constants $C_{1}$ and $C_{2}$ independent of $T$ and $\mathscr{T}_{j}$. $d(T)$ denotes the diameter of $T$ and $\phi_{\gamma}$ is given by (2.3).

If $T$ is a triangle from triangulation $\mathscr{T}_{j}$, and $\phi_{\gamma}(x)$ vanishes for a point $x \in T$, the estimate

$$
C_{1} 2^{-j} \max _{x \in T} \phi_{\gamma}(x) \leqslant d(T) \leqslant C_{2} 2^{-j} \max _{x \in T} \phi_{\gamma}(x)
$$

holds with the same constants $C_{1}$ and $C_{2}$ as in (2.4).

Babuška, Kellogg, and Pitkäranta [2] have examined the approximation properties of finite-element spaces based on such triangulations. To describe their results, we have to define some additional norms:

Let

$$
\|u\|_{0 ; \gamma}=\left(\int \phi_{\gamma}^{2}(x)|u(x)|^{2} d x\right)^{1 / 2}
$$


be a weighted $L_{2}$-norm and $W^{0,2 ; \gamma}(\Omega)$ be the space of all measurable functions on $\Omega$ for which this norm is finite. Define $\phi_{-\gamma}$ as

$$
\phi_{-\gamma}(x)=\prod_{i=1}^{n}\left|x-x_{i}\right|^{-\gamma_{i}}
$$

and the norm \|\|$_{0 ;-\gamma}$ on $W^{0,2 ;-\gamma}(\Omega)$ analogously to (2.6), with $\phi_{\gamma}$ replaced by $\phi_{-\gamma}$. Let $W^{2,2 ; \gamma}(\Omega)$ be the space of all functions $u \in W^{1,2}(\Omega)$ with

$$
\|u\|_{2 ; \gamma}=\left(\|u\|_{1}^{2}+\sum_{|\alpha|=2}\left\|D^{\alpha} u\right\|_{0, \gamma}^{2}\right)^{1 / 2}
$$

being finite. Using Hölder's inequality and $H^{1}(\Omega) \subseteq L^{p}(\Omega), 1 \leqslant p<\infty$, it can be shown that $H^{1}(\Omega)$ is continuously imbedded in $W^{0,2 ;-\gamma}(\Omega)$. Therefore, the linear functional defined by (1.2) is a continuous linear functional on $H_{D}^{1}(\Omega)$, if the function $f$ belongs to the weighted space $W^{0,2 ; \gamma}(\Omega)$. For $f \in W^{0,2 ; \gamma}(\Omega)$, the boundary value problem (1.1), (1.3), therefore, has a uniquely determined solution $u \in H_{D}^{1}(\Omega)$ depending continuously on $f$. Its regularity properties are derived in [9]; see also [2].

THEOREM 2.2. If the coefficients $\gamma_{i}$ are chosen as above, and $f$ is taken from $W^{0.2 ; \gamma}(\Omega)$, the solution $u$ of the boundary value problem (1.1), (1.3) belongs to $W^{2,2: \gamma}(\Omega)$, and there is a constant $C$ independent of $f$ such that

$$
\|u\|_{2 ; \gamma} \leqslant C\|f\|_{0 ; \gamma} .
$$

Babuška, Kellogg, and Pitkäranta [2] have shown:

THEOREM 2.3. For all functions $u \in W^{2,2 ; \gamma}(\Omega)$, the estimate

$$
\left\|u-P_{j} u\right\|_{1} \leqslant C h_{j}\|u\|_{2 ; \gamma}
$$

holds, with $h_{j}=2^{-j}$ and a constant $C$ independent of $u$ and $j$.

Of course, the constant in Theorem 2.3 depends on the properties of the family of triangulations and on the choice of $\gamma$, but not on the regularity theory of the boundary value problem.

Using a duality argument, one is now able to prove the approximation property of the finite-element spaces needed for our discussion of multi-level methods. This approximation property is an extension of the well-known $L_{2}$-norm estimate of the error for convex domains:

TheOrem 2.4 (see [2, TheOrem 5.2]). For all functions $u \in H_{D}^{1}(\Omega)$, the estimate

$$
\left\|u-P_{j} u\right\|_{0 ;-\gamma} \leqslant C h_{j}\left\|P_{j} u-u\right\|_{1}
$$

holds, with $h_{j}=2^{-j}$ and a constant $C$ independent of $u$ and $j$.

From the result stated in Theorem 2.4 we can derive the approximation property (1.13):

THEOREM 2.5. There exists a constant $K$ independent of $j \geqslant 1$ such that

$$
\left\|u-P_{j-1} u\right\|_{j} \leqslant K\left\|u-P_{j-1} u\right\|_{1}
$$

for all functions $u \in S_{D}\left(\mathscr{T}_{j}\right)$. 
Proof. Under the given assumptions on the family of triangulations there are constants $C_{1}$ and $C_{2}$ independent of $j$ such that

$$
\begin{aligned}
\left\|u-P_{j-1} u\right\|_{j}^{2} & =\sum_{T \in \mathscr{T}_{j}} \sum_{x \in \mathscr{N}_{j} \cap T}\left|\left(u-P_{j-1} u\right)(x)\right|^{2} \\
& \leqslant C_{1} \sum_{T \in \mathscr{T}_{j}} d(T)^{-2} \int_{T}\left|\left(u-P_{j-1} u\right)(x)\right|^{2} d x \\
& \leqslant C_{2} \sum_{T \in \mathscr{T}_{j}} h_{j}^{-2} \int_{T} \phi_{\gamma}^{-2}(x)\left|\left(u-P_{j-1} u\right)(x)\right|^{2} d x \\
& =C_{2} h_{j}^{-2}\left\|u-P_{j-1} u\right\|_{0 ;-\gamma}^{2}
\end{aligned}
$$

for all functions $u \in S_{D}\left(\mathscr{T}_{j}\right)$. Using Theorem 2.4 and $h_{j-1} / h_{j}=2$, we get the assertion.

3. A Convergence Proof for the Multi-Level Method. We begin with an analysis of the effect of the smoothing steps (1.12):

Lemma 3.1. If $K_{1}$ is the constant of Theorem 2.1 and $0<\omega \leqslant 1 / K_{1}^{2}$, and if the approximations $u$ are defined as in (1.10), (1.11), and (1.12), we have the estimate

$$
\left\|P_{j} u-u_{m+1}\right\|_{1} \leqslant \frac{1}{\sqrt{\omega}} \frac{1}{\sqrt{2 m+1}}\left\|P_{j} u-u_{1}\right\|_{j} .
$$

Proof. We use a basis $\Psi_{1}, \ldots, \Psi_{N}$ of $S_{D}\left(\mathscr{T}_{j}\right)$ with

$$
E_{j}\left(\Psi_{i}, \Psi_{k}\right)=\delta_{i k}, \quad B\left(\Psi_{i}, \Psi_{k}\right)=\lambda_{i} \delta_{i k} .
$$

If $P_{j} u-u_{1}$ is given by

$$
P_{j} u-u_{1}=\sum_{i=1}^{N} c_{i} \Psi_{i}
$$

we get from (1.12) for $l=0, \ldots, m$,

$$
P_{j} u-u_{l+1}=\sum_{i=1}^{N}\left(1-\omega \lambda_{i}\right)^{l} c_{i} \Psi_{i}
$$

and therefore

$$
\left\|P_{j} u-u_{m+1}\right\|_{1}^{2}=\frac{1}{\omega} \sum_{i=1}^{N} \omega \lambda_{i}\left(1-\omega \lambda_{i}\right)^{2 m} c_{i}^{2} .
$$

Because of Theorem 2.1 we have $0<\lambda_{i} \leqslant K_{1}^{2}$. Thus for $0<\omega \leqslant 1 / K_{1}^{2}$ the estimate $0<\omega \lambda_{i} \leqslant 1$ holds. An elementary discussion of $g(x)=x(1-x)^{2 m}$ in the interval $0 \leqslant x \leqslant 1$ now gives

$$
\begin{aligned}
\left\|P_{j} u-u_{m+1}\right\|_{1}^{2} & \leqslant \frac{1}{\omega} \frac{1}{2 m+1}\left(1-\frac{1}{2 m+1}\right)^{2 m} \sum_{i=1}^{N} c_{i}^{2} \\
& =\frac{1}{\omega} \frac{1}{2 m+1}\left(1-\frac{1}{2 m+1}\right)^{2 m}\left\|P_{j} u-u_{1}\right\|_{j}^{2} .
\end{aligned}
$$

For the optimal choice $\omega=1 / K_{1}^{2},(3.1)$ reduces to

$$
\left\|P_{j} u-u_{m+1}\right\|_{1} \leqslant \frac{K_{1}}{\sqrt{2 m+1}}\left\|P_{j} u-u_{1}\right\|_{j} .
$$


By Theorem 2.1, $K_{1}$ is bounded independently of $j$, and this justifies the name "smoothing property" for (1.14).

With the help of Lemma 3.1 and Theorem 2.5, we are able to prove the convergence of the two-level procedure:

THEOREM 3.1. If $\omega$ is chosen such that the estimate $0<\omega \leqslant 1 / K_{1}^{2}$ is satisfied with the constant $K_{1}$ from the smoothing property (1.14) and Theorem 2.1, respectively, and if the approximations $u_{l}$ of $P_{j} u$ are defined as in (1.10), (1.11), and (1.12), the estimate

$$
\left\|P_{j} u-u_{m+1}\right\|_{1} \leqslant \frac{K}{\sqrt{\omega}} \frac{1}{\sqrt{2 m+1}}\left\|P_{j} u-u_{0}\right\|_{1}
$$

holds. $K$ is the constant from the approximation property and Theorem 2.4 , respectively. Thus each step of the two-level procedure reduces the energy norm of the error by a factor behaving like $O(1 / \sqrt{m})$ for a growing number $m$ of smoothing steps, if the parameter $\omega$ is chosen as above.

Proof. By definition of $u_{1}$,

$$
P_{j} u-u_{1}=\left(P_{j} u-u_{0}\right)-P_{j-1}\left(P_{j} u-u_{0}\right)
$$

holds and, with the help of Theorem 2.5, we get

$$
\left\|P_{j} u-u_{1}\right\|_{j} \leqslant K\left\|P_{j} u-u_{1}\right\|_{1} \leqslant K\left\|P_{j} u-u_{0}\right\|_{1} .
$$

This estimate and (3.1) lead to the desired result.

The optimal choice $\omega=1 / K_{1}^{2}$ gives

$$
\left\|P_{j} u-u_{m+1}\right\|_{1} \leqslant \frac{K K_{1}}{\sqrt{2 m+1}}\left\|P_{j} u-u_{0}\right\|_{1} .
$$

The convergence of the full multi-level process with nearly the same speed of convergence is a consequence of the preceding theorem:

THEOREM 3.2. One step of the full multi-level procedure reduces the energy norm of the error by a factor behaving like $O(1 / \sqrt{m})$ for a growing number $m$ of smoothing steps per level, uniformly in the number of levels, if the parameter $\omega$ in the smoothing steps is chosen as in Theorem 3.1.

Proof. The assertion is proved by induction over the number $j$ of levels. Assume that one step of the $j$-level method reduces the energy norm of the error at least by the factor $\delta_{j}$.

In the multi-level iteration the correction term $d \in S_{D}\left(\mathscr{T}_{j-1}\right)$ in (1.11) is replaced by an approximate correction $\tilde{d} \in S_{D}\left(\mathscr{T}_{j-1}\right)$. Let $\tilde{u}_{1}=u_{0}+\tilde{d}$ and $\tilde{u}_{2}, \ldots, \tilde{u}_{m+1}$ be defined analogously to (1.12), with $u_{l}$ replaced by $\tilde{u}_{l}$. If $u_{1}-\tilde{u}_{1}$ has the representation

$$
u_{1}-\tilde{u}_{1}=\sum_{i=1}^{N} a_{i} \Psi_{i}
$$


in the basis from the proof of Lemma 3.1, the final error is given by

$$
u_{m+1}-\tilde{u}_{m+1}=\sum_{i=1}^{N}\left(1-\omega \lambda_{i}\right)^{m} a_{i} \Psi_{i} .
$$

Using $0<\omega \lambda_{i} \leqslant 1$, we therefore get

$$
\left\|u_{m+1}-\tilde{u}_{m+1}\right\|_{1} \leqslant\left\|u_{1}-\tilde{u}_{1}\right\|_{1}=\|d-\tilde{d}\|_{1} .
$$

$\tilde{d}$ is the approximate solution of (1.10) produced by $p$ steps of the $(j-1)$-level method, with $d_{0}=0$ as starting value. As the exact solution $d$ of (1.10) is given by $d=P_{j-1}\left(P_{j} u-u_{0}\right)$, we have

$$
\|d-\tilde{d}\|_{1} \leqslant \delta_{j-1}^{p}\left\|P_{j-1}\left(P_{j} u-u_{0}\right)\right\|_{1},
$$

and therefore

$$
\left\|u_{m+1}-\tilde{u}_{m+1}\right\|_{1} \leqslant \delta_{j-1}^{p}\left\|P_{j} u-u_{0}\right\|_{1} .
$$

By Theorem 3.1, the estimate

$$
\left\|P_{j} u-u_{m+1}\right\|_{1} \leqslant \frac{K}{\sqrt{\omega}} \frac{1}{\sqrt{2 m+1}}\left\|P_{j} u-u_{0}\right\|_{1}
$$

holds. Together with the estimate proved before, we get

$$
\left\|P_{j} u-\tilde{u}_{m+1}\right\|_{1} \leqslant\left\{\delta_{j-1}^{p}+\frac{K}{\sqrt{\omega}} \frac{1}{\sqrt{2 m+1}}\right\}\left\|P_{j} u-u_{0}\right\|_{1},
$$

which gives the final estimate

$$
\delta_{j} \leqslant \delta_{j-1}^{p}+\frac{K}{\sqrt{\omega}} \frac{1}{\sqrt{2 m+1}}
$$

for $\delta_{j}$. If we choose $m$ such that

$$
\frac{K}{\sqrt{\omega}} \frac{1}{\sqrt{2 m+1}} \leqslant \frac{1}{4}
$$

is satisfied, because of $\delta_{0}=0$ and $p \geqslant 2$ the $\delta_{j}$ 's are majorized by the smaller one of the two roots $\delta$ of the equation

$$
\delta=\delta^{2}+\frac{K}{\sqrt{\omega}} \frac{1}{\sqrt{2 m+1}} .
$$

This root can be estimated by

$$
2 \frac{K}{\sqrt{\omega}} \frac{1}{\sqrt{2 m+1}}
$$

which completes the proof.

Under the assumptions (2.4) and (2.5) on the triangulations, we have the estimate

$$
C_{1} 4^{j} \leqslant N_{j} \leqslant C_{2} 4^{j}
$$

for the number $N_{j}$ of nodes of the triangulation $\mathscr{T}_{j}$ with positive constants $C_{1}$ and $C_{2}$ independent of $j$. Because of this estimate, the number of computer operations necessary to realize a single iteration step of the $j$-level procedure can be estimated in terms of the number of unknowns in the $j$ th level: If $F_{j-1}$ is the number of 
operations necessary to realize one step of the $(j-1)$-level method, we get analogously to [4] the estimate

$$
F_{j} \leqslant p F_{j-1}+C m N_{j}
$$

for the cost $F_{j}$ of one step of the $j$-level method. The first term on the right-hand side of (3.6) represents the amount of work necessary for computing the correction term (1.10) by $p$ steps of the $(j-1)$-level method, and the second term, the work done in the $m$ smoothing steps. Using (3.5) and (3.6), for $p=2$ and $p=3$, we get an estimate

$$
L_{1} m N_{j} \leqslant F_{j} \leqslant L_{2} m N_{j}
$$

with positive constants $L_{1}$ and $L_{2}$ independent of $j$ and $m$. That means that the amount of work necessary to reduce the error of an initial approximation by a constant factor is proportional to the number of unknowns of the problem.

For the exact solution $u$ of the boundary value problem (1.1), (1.3) and its finite-element projection $P_{j} u$ on $S_{D}\left(\mathscr{T}_{j}\right)$, we have by Theorems 2.2 and 2.3,

$$
\left\|u-P_{j} u\right\|_{1} \leqslant C h_{j}\|u\|_{2 ; \gamma} \leqslant C h_{j}\|f\|_{0 ; \gamma} .
$$

Moreover, using the nested iteration process described in [4] and [8], it is therefore possible to get an approximation for $P_{j} u$ of order $O\left(h_{j}\right)$ with an amount of work proportional to the number of unknowns in the $j$ th level.

Note Added in Proof. After the completion of this paper in 1983 many convergence proofs based on algebraic properties like our approximation and smoothing property have been given. As examples we mention the papers of Bank and Douglas [11] and the author [12]. In these papers it is proved that "symmetrized" multi-level methods converge for every number of smoothing steps per level, also in the case of the $V$-cycle. Using the results obtained in Section 2, one can apply these new theories to the situation considered here.

Fachbereich Mathematik

Universität Dortmund

Vogelpothsweg 87

D-4600 Dortmund 50

Federal Republic of Germany

1. G. P. Astrahancev, “An iterative method for solving elliptic net problems,” Ž. Vyčisl. Mat. i Mat. Fiz., v. 11, 1971, 439-448. (Russian)

2. I. BABUSKA, R. B. Kellogg \& J. PitKäranta, "Direct and inverse error estimates for finite elements with mesh refinements," Numer. Math., v. 33, 1979, pp. 447-471.

3. N. S. BAHVALOV, "On the convergence of a relaxation method with natural constraints on the elliptic operator,” Ž. Vyčisl. Mat. i. Mat. Fiz., v. 6, 1966, pp. 861-883. (Russian)

4. R. E. BanK \& T. Dupont, "An optimal order process for solving finite element equations," Math. Comp., v. 36, 1981, pp. 35-51.

5. R. E. Bank \& A. H. Sherman, "An adaptive multi-level method for elliptic boundary value problems," Computing, v. 26, 1981, pp. 91-105.

6. A. BRANDT, "Multi-level adaptive solution to boundary value problems," Math. Comp., v. 31, 1977, pp. 333-390.

7. R. P. Fedorenko, "A relaxation method for solving elliptic difference equations," Ž. Vyčisl. Mat. $i$ Mat. Fiz., v. 1, 1961, pp. 922-927. (Russian); "The speed of convergence of an iterative process," $\check{Z}$. Vyčisl. Mat. i Mat. Fiz., v. 4, 1964, pp. 559-564. (Russian) 
8. W. НАСКвUSCH, “On the convergence of multi-grid iterations," Beiträge Numer. Math., v. 9, 1981, pp. $213-329$.

9. V. A. KondRAT'EV, “Boundary problems for elliptic equations in domains with conical or angular points," Trans. Moscow Math. Soc., v. 16, 1967, pp. 227-313.

10. R. A. Nicolaides, "On the $l^{2}$ convergence of an algorithm for solving finite element equations," Math. Comp., v. 31, 1977, pp. 892-906.

11. R. E. Bank \& C. C. Douglas, "Sharp estimates for multigrid rates of convergence with general smoothing and approximation," SIAM J. Numer. Anal., v. 22, 1985, pp. 617-633.

12. H. YSERENTANT, "On the convergence of multi-level methods for strongly nonuniform families of grids and any number of smoothing steps per level," Computing, v. 30, 1983, pp. 305-313. 